\title{
Existence of Nonoscillatory Solutions of Higher-Order Neutral Differential Equations with Distributed Coefficients and Delays
}

\author{
Youjun Liu, ${ }^{1}$ Huanhuan Zhao, ${ }^{1}$ and Jurang Yan $^{2}$ \\ ${ }^{1}$ College of Mathematics and Computer Sciences, Shanxi Datong University, Datong, Shanxi 037009, China \\ ${ }^{2}$ School of Mathematical Sciences, Shanxi University, Taiyuan, Shanxi 030006, China \\ Correspondence should be addressed to Youjun Liu; lyj9791@126.com
}

Received 23 May 2016; Revised 11 July 2016; Accepted 12 July 2016

Academic Editor: Massimiliano Ferrara

Copyright (c) 2016 Youjun Liu et al. This is an open access article distributed under the Creative Commons Attribution License, which permits unrestricted use, distribution, and reproduction in any medium, provided the original work is properly cited.

We consider the existence of nonoscillatory solutions of higher-order neutral differential equations with distributed coefficients. We use the Banach contraction principle to obtain new sufficient condition for the existence of nonoscillatory solutions.

\section{Introduction}

In this paper, we consider the higher-order nonlinear neutral differential equation with distributed deviating arguments:

$$
\begin{gathered}
{\left[\Phi\left[r(t) x(t)+\int_{a}^{b} p(t, \theta) x(t-\theta) d \theta\right]\right]^{(n)}} \\
\quad+\int_{c}^{d} q_{1}(t, \tau) g_{1}(x(t-\tau)) d \tau \\
\quad-\int_{e}^{f} q_{2}(t, \sigma) g_{2}(x(t-\sigma)) d \sigma=h(t) .
\end{gathered}
$$

Throughout this paper, the following conditions are assumed to hold:

(1) where $n \geq 1$ is a positive integer, $r \in C\left(\left[t_{0}\right.\right.$, $\left.\infty), R^{+}\right), 0<a<b, 0<c<d, 0<e<f$;

(2) $p \in C\left(\left[t_{0}, \infty\right) \times[a, b], R\right), q_{1} \in C\left(\left[t_{0}, \infty\right) \times[c, d]\right.$, $\left.R^{+}\right), q_{2} \in C\left(\left[t_{0}, \infty\right) \times[e, f], R^{+}\right), h \in C\left(\left[t_{0}, \infty\right), R\right) ;$

(3) $\Phi(u)$ are continuously increasing real odd function with respect to $u$ defined on $R$, and $\Phi^{-1}(u)$ satisfy the local Lipschitz condition;

(4) $g_{i} \in C(R, R), g_{i}(u)$ satisfy the local Lipschitz condition and $u g_{i}(u)>0, i=1,2$, for $u \neq 0$.

Recently there have been a lot of activities concerning the existence of nonoscillatory solutions for neutral differential equations with positive and negative coefficient. In 2002,
Zhou and Zhang [1] studied higher-order linear neutral delay differential equation:

$$
\begin{aligned}
& \frac{d^{n}}{d t^{n}}[x(t)+c x(t-\tau)] \\
& \quad+(-1)^{n+1}[P(t) x(t-\sigma)-Q(t) x(t-\delta)]=0 .
\end{aligned}
$$

In 2005, the existence of nonoscillatory solutions of firstorder linear neutral delay differential equations of the form

$$
\begin{gathered}
\frac{d}{d t}[x(t)+P(t) x(t-\tau)]+Q_{1}(t) x\left(t-\sigma_{1}\right) \\
-Q_{2}(t) x\left(t-\sigma_{2}\right)=0
\end{gathered}
$$

was investigated by Zhang et al. [2] and, in the same year, Yu and Wang [3] studied nonoscillatory solutions of secondorder nonlinear neutral differential equations of the form

$$
\begin{aligned}
& {\left[r(t)[x(t)+P(t) x(t-\tau)]^{\prime}\right]^{\prime}+Q_{1}(t) f\left(x\left(t-\sigma_{1}\right)\right)} \\
& -Q_{2}(t) g\left(x\left(t-\sigma_{2}\right)\right)=0 .
\end{aligned}
$$

In 2010, Candan and Dahiya [4] studied nonoscillatory solutions of first-order and second-order nonlinear neutral differential equations with positive and negative coefficients:

$$
\begin{gathered}
\frac{d^{k}}{d t^{k}}[x(t)+P(t) x(t-\tau)]+\int_{a}^{b} q_{1}(t, \xi) x(t-\xi) d \xi \\
-\int_{c}^{d} q_{2}(t, \mu) x(t-\mu) d \mu=0, \quad k=1,2 .
\end{gathered}
$$


In 2012, Candan [5] study higher-order nonlinear differential equation:

$$
\begin{aligned}
& {\left[r(t)[x(t)+P(t) x(t-\tau)]^{(n-1)}\right]^{\prime}+(-1)^{n}} \\
& \cdot\left[Q_{1}(t) g_{1}\left(x\left(t-\sigma_{1}\right)\right)-Q_{2}(t) g_{2}(x(t-\mu))\right. \\
& \quad-f(t)]=0 .
\end{aligned}
$$

In 2015, Liu et al. [6] study higher-order neutral differential equation with distributed deviating arguments:

$$
\begin{aligned}
{[r(t)} & x(t)+P(t) x(t-\theta)]^{(n)} \\
& +\int_{c}^{d} q_{1}(t, \tau) g_{1}(x(t-\tau)) d \tau \\
& -\int_{e}^{f} q_{2}(t, \sigma) g_{2}(x(t-\sigma)) d \sigma=f(t) .
\end{aligned}
$$

As can be seen from the development process of the above equations, the delay of neutral part in the discussed differential equations was all constant delays, and the main thought in the employed verification method also kept the same in which the coefficient neutral part in the neutral was all discussed in four cases, that is, $(-\infty,-1),(-1,0),(0,1)$, and $(1,+\infty)$, and then was verified by constructing the corresponding operator. However, the case for distributed deviating arguments is rather rare. In 2013, Candan [7] studied first-order neutral differential equation with distributed deviating arguments:

$$
\begin{aligned}
& {\left[\left[x(t)-\int_{a}^{b} p_{2}(t, \xi) x(t-\xi) d \xi\right]^{\gamma}\right]^{\prime}} \\
& \quad+\int_{c}^{d} Q_{2}(t, \xi) G(x(t-\xi)) d \xi=0,
\end{aligned}
$$

where $\gamma$ is a ratio of odd positive integers; however the discussion only covered the condition for coefficient being $0<\int_{a}^{b} p_{2}(t, \xi) d \xi<1$, while being without the other three conditions, which might be caused by the difficulty in establishing feasible operator. In view of the above, here, in this paper, the difficulty of operator establishment was settled and sufficient condition for the existence of nonoscillatory solutions of differential equation with coefficient of $\int_{a}^{b} p_{2}(t, \xi) d \xi$ in the four cases was obtained. Thus, this paper may present its theoretical value as well as practical application value. For related work, we refer the reader to the references [8-12].

As usual, a solution of (1) is said to be oscillatory if it has arbitrarily large zeros. Otherwise the solution is said to be nonoscillatory.
A solution of (1) is a continuous function $x(t)$ defined on $\left[t_{1}-\mu, \infty\right)$, for some $t_{1}>t_{0}$, such that $\Phi(r(t) x(t)+$ $\left.\int_{a}^{b} p(t, \theta) x(t-\theta) d \theta\right)$ is $n$ times continuously differentiable and (1) holds for all $n \geq 1$. Here, $\mu=\max \{b, d, f\}$.

Let $L_{i}, i=1,2$ denote the Lipschitz constants of functions $g_{i}, i=1,2$ on the set $A, K$ denote the Lipschitz constants of functions $\Phi^{-1}(u)$, respectively, and $L=$ $\max \left\{L_{1}, L_{2}\right\}, \beta_{i}=\max _{x \in A} g_{i}(x), i=1,2$.

\section{The Main Results}

Theorem 1. Assume that $0 \leq \int_{a}^{b} p(t, \theta) d \theta \leq p_{1}<1,1 \leq$ $r(t) \leq 1 / p_{1}$, and

$$
\begin{array}{r}
\int_{t}^{\infty} s^{n-1} \int_{c}^{d} q_{1}(s, \tau) d \tau d s<\infty \\
\int_{t}^{\infty} s^{n-1} \int_{e}^{f} q_{2}(s, \sigma) d \sigma d s<\infty \\
\int_{t}^{\infty} s^{n-1} h(s) d s<\infty .
\end{array}
$$

Then (1) has a bounded nonoscillatory solution.

Proof. Let $\Lambda$ be the set of all continuous and bounded functions on $\left[t_{0}, \infty\right)$ and the norm be $\|x(t)\|=\sup _{t_{0} \leq t<+\infty} x(t)$. Set $A=\left\{x \in \Lambda, M_{1} \leq x(t) \leq M_{2}, t \geq t_{0}\right\}$, where $M_{1}, M_{2}$ are two positive constants such that $p_{1} M_{2}+M_{1} / p_{1}<\alpha<M_{2}$. From (9), one can choose $t_{1} \geq t_{0}+\mu$ and sufficiently large $t \geq t_{1}$ such that

$$
\begin{aligned}
& \int_{t}^{\infty} \frac{(s-t)^{n-1}}{(n-1) !}\left[\beta_{1} \int_{c}^{d} q_{1}(s, \tau) d \tau+|h(s)|\right] d s \\
& \quad \leq \Phi\left(M_{2}\right)-\Phi(\alpha) \\
& \int_{t}^{\infty} \frac{(s-t)^{n-1}}{(n-1) !}\left[\beta_{2} \int_{e}^{f} q_{2}(s, \sigma) d \sigma+|h(s)|\right] d s \\
& \quad \leq \Phi(\alpha)-\Phi\left(p_{1} M_{2}+\frac{M_{1}}{p_{1}}\right), \\
& \int_{t}^{\infty} \frac{(s-t)^{n-1}}{(n-1) !}\left[\int_{c}^{d} q_{1}(s, \tau) d \tau+\int_{e}^{f} q_{2}(s, \sigma) d \sigma\right] d s \\
& \leq \frac{1-p_{1}}{K L},
\end{aligned}
$$

and define an operator $T$ on $A$ as follows:

$$
\begin{aligned}
& (T x)(t) \\
& =\left\{\begin{array}{l}
\frac{1}{r(t)}\left\{-\int_{a}^{b} p(t, \theta) x(t-\theta) d \theta+\Phi^{-1}\left[\Phi(\alpha)+\int_{t}^{\infty} \frac{(s-t)^{n-1}}{(n-1) !}\left[\int_{c}^{d} q_{1}(s, \tau) g_{1}(x(s-\tau)) d \tau-\int_{e}^{f} q_{2}(s, \sigma) g_{2}(x(s-\sigma)) d \sigma-h(s)\right] d s\right]\right\}, \quad t \geq t_{1}, \quad t_{0} \leq t \leq t_{1} \\
(T x)\left(t_{1}\right),
\end{array}\right.
\end{aligned}
$$


It is easy to see that $T$ is continuous, for $t \geq t_{1}, x \in A$. By using (10), we have

$$
\begin{aligned}
& (T x)(t) \leq \frac{1}{r(t)}\left\{\Phi^{-1}[\Phi(\alpha)\right. \\
& \left.\left.+\int_{t}^{\infty} \frac{(s-t)^{n-1}}{(n-1) !}\left[\int_{c}^{d} q_{1}(s, \tau) g_{1}(x(s-\tau)) d \tau-h(s)\right] d s\right]\right\} \\
& \leq \Phi^{-1}[\Phi(\alpha) \\
& \left.+\int_{t}^{\infty} \frac{(s-t)^{n-1}}{(n-1) !}\left(\beta_{1} \int_{c}^{d} q_{1}(s, \tau) d \tau+|h(s)|\right) d s\right] \\
& \leq \Phi^{-1}\left[\Phi(\alpha)+\Phi\left(M_{2}\right)-\Phi(\alpha)\right]=M_{2},
\end{aligned}
$$

and, taking (11) into account, we have

$$
(T x)(t) \geq \frac{1}{r(t)}\left\{-\int_{a}^{b} p(t, \theta) x(t-\theta) d \theta-\Phi^{-1}[\Phi(\alpha)\right.
$$

$$
\begin{aligned}
& \left.\left.-\int_{t}^{\infty} \frac{(s-t)^{n-1}}{(n-1) !}\left[\int_{e}^{f} q_{2}(s, \sigma) g_{2}(x(s-\sigma)) d \sigma+h(s)\right] d s\right]\right\} \\
& \geq p_{1}\left\{-p_{1} M_{2}+\Phi^{-1}[\Phi(\alpha)\right. \\
& \left.\left.-\int_{t}^{\infty} \frac{(s-t)^{n-1}}{(n-1) !}\left(\beta_{2} \int_{e}^{f} q_{2}(s, \sigma) d \sigma+|h(s)|\right) d s\right]\right\} \\
& \geq p_{1}\left\{-p_{1} M_{2}+\Phi^{-1}\left[\Phi(\alpha)-\Phi(\alpha)+\Phi\left(p_{1} M_{2}+\frac{M_{1}}{p_{1}}\right)\right]\right\} \\
& =M_{1} .
\end{aligned}
$$

These show that $T A \subset A$. Since $A$ is bounded, close, convex subset of $\Lambda$, in order to apply the contraction principle we have to show that $T$ is a contraction mapping on $A$. For $\forall x_{1}, x_{2} \in A$, and $t \geq t_{1}$,

$$
\begin{aligned}
& \left|\left(T x_{1}\right)(t)-\left(T x_{2}\right)(t)\right| \leq \frac{1}{r(t)}\left\{\int_{a}^{b} p(t, \theta)\left|x_{1}(t-\theta)-x_{2}(t-\theta)\right| d \theta+\Phi^{-1}[\Phi(\alpha)\right. \\
& \left.\quad+\int_{t}^{\infty} \frac{(s-t)^{n-1}}{(n-1) !}\left|\int_{c}^{d} q_{1}(s, \tau) g_{1}\left(x_{1}(s-\tau)\right) d \tau-\int_{e}^{f} q_{2}(s, \sigma) g_{2}\left(x_{1}(s-\sigma)\right) d \sigma-h(s)\right| d s\right]-\Phi^{-1}[\Phi(\alpha) \\
& \left.\left.\quad+\int_{t}^{\infty} \frac{(s-t)^{n-1}}{(n-1) !}\left|\int_{c}^{d} q_{1}(s, \tau) g_{1}\left(x_{2}(s-\tau)\right) d \tau-\int_{e}^{f} q_{2}(s, \sigma) g_{2}\left(x_{2}(s-\sigma)\right) d \sigma-h(s)\right| d s\right]\right\} \leq \frac{1}{r(t)}\left\{p_{1}\left|x_{1}-x_{2}\right|\right. \\
& \quad+K \int_{t}^{\infty} \frac{(s-t)^{n-1}}{(n-1) !}\left[\int_{c}^{d} q_{1}(s, \tau)\left|g_{1}\left(x_{1}(s-\tau)\right)-g_{1}\left(x_{2}(s-\tau)\right)\right| d \tau\right. \\
& \left.\left.\quad+\int_{e}^{f} q_{2}(s, \sigma)\left|g_{2}\left(x_{1}(s-\sigma)\right)-g_{2}\left(x_{2}(s-\sigma)\right)\right| d \sigma\right] d s\right\} .
\end{aligned}
$$

Using (12),

$$
\begin{gathered}
\left|\left(T x_{1}\right)(t)-\left(T x_{2}\right)(t)\right| \leq\left|x_{1}-x_{2}\right|\left(p_{1}\right. \\
+K L \int_{t}^{\infty} \frac{(s-t)^{n-1}}{(n-1) !}\left[\int_{c}^{d} q_{1}(s, \tau) d \tau\right. \\
\left.\left.\quad+\int_{e}^{f} q_{2}(s, \sigma) d \sigma\right] d s\right)<\rho\left|x_{1}-x_{2}\right| .
\end{gathered}
$$

This implies with the sup norm that

$$
\left\|T x_{1}-T x_{2}\right\|<\rho\left\|x_{1}-x_{2}\right\|,
$$

where $\rho=1$, which shows that $T$ is a contraction mapping on $A$ and therefore there exists a unique solution, obviously a bounded positive solution of (1) $x \in A$, such that Tx $=x$. The proof is complete.

Theorem 2. Assume that $1<p_{3} \leq \int_{a}^{b} p(t, \theta) d \theta \leq p_{2}<2 p_{3}<$ $+\infty, 2 p_{3} \leq r(t) \leq 2 p_{2}$ and that (9) holds.

Then (1) has a bounded nonoscillatory solution.
Proof. Let $\Lambda$ be the set of all continuous and bounded functions on $\left[t_{0}, \infty\right)$ and the norm be $\|x(t)\|=\sup _{t_{0} \leq t<+\infty} x(t)$. Set $A=\left\{x \in \Lambda, M_{3} \leq x(t) \leq M_{4}, t \geq t_{0}\right\}$, where $M_{3}, M_{4}$ are two positive constants such that $p_{2} M_{4}+2 p_{2} M_{3}<\alpha<2 p_{3} M_{4}$. From (9), one can choose $t_{1} \geq t_{0}+\mu$ and sufficiently large $t \geq t_{1}$ such that

$$
\begin{aligned}
& \int_{t}^{\infty} \frac{(s-t)^{n-1}}{(n-1) !}\left[\beta_{1} \int_{c}^{d} q_{1}(s, \tau) d \tau+|h(s)|\right] d s \\
& \quad \leq \Phi\left(2 p_{3} M_{4}\right)-\Phi(\alpha) \\
& \int_{t}^{\infty} \frac{(s-t)^{n-1}}{(n-1) !}\left[\beta_{2} \int_{e}^{f} q_{2}(s, \sigma) d \sigma+|h(s)|\right] d s \\
& \quad \leq \Phi(\alpha)-\Phi\left(p_{2} M_{4}-2 p_{2} M_{3}\right), \\
& \int_{t}^{\infty} \frac{(s-t)^{n-1}}{(n-1) !}\left[\int_{c}^{d} q_{1}(s, \tau) d \tau+\int_{e}^{f} q_{2}(s, \sigma) d \sigma\right] d s \\
& \leq \frac{2 p_{3}-p_{2}}{K L},
\end{aligned}
$$


and define an operator $T$ on $A$ as follows:

$(T x)(t)$

$=\left\{\begin{array}{l}\frac{1}{r(t)}\left\{-\int_{a}^{b} p(t, \theta) x(t-\theta) d \theta+\Phi^{-1}\left[\Phi(\alpha)+\int_{t}^{\infty} \frac{(s-t)^{n-1}}{(n-1) !}\left[\int_{c}^{d} q_{1}(s, \tau) g_{1}(x(s-\tau)) d \tau-\int_{e}^{f} q_{2}(s, \sigma) g_{2}(x(s-\sigma)) d \sigma-h(s)\right] d s\right]\right\}, \quad t \geq t_{1}, \\ (T x)\left(t_{1}\right),\end{array}\right.$

It is easy to see that $T$ is continuous, for $t \geq t_{1}, x \in A$. By using (19), we have

$$
\begin{aligned}
& (T x)(t) \leq \frac{1}{r(t)}\left\{\Phi^{-1}[\Phi(\alpha)\right. \\
& \left.\left.+\int_{t}^{\infty} \frac{(s-t)^{n-1}}{(n-1) !}\left[\int_{c}^{d} q_{1}(s, \tau) g_{1}(x(s-\tau)) d \tau-h(s)\right] d s\right]\right\} \\
& \quad \leq \frac{1}{2 p_{3}}\left\{\Phi^{-1}[\Phi(\alpha)\right. \\
& \left.\left.+\int_{t}^{\infty} \frac{(s-t)^{n-1}}{(n-1) !}\left[\beta_{1} \int_{c}^{d} q_{1}(s, \tau) d \tau+|h(s)|\right] d s\right]\right\} \leq M_{4}
\end{aligned}
$$

$$
\begin{aligned}
& \left|\left(T x_{1}\right)(t)-\left(T x_{2}\right)(t)\right| \leq \frac{1}{r(t)}\left\{\int_{a}^{b} p(t, \theta)\left|x_{1}(t-\theta)-x_{2}(t-\theta)\right| d \theta+\Phi^{-1}[\Phi(\alpha)\right. \\
& \left.\quad+\int_{t}^{\infty} \frac{(s-t)^{n-1}}{(n-1) !}\left|\int_{c}^{d} q_{1}(s, \tau) g_{1}\left(x_{1}(s-\tau)\right) d \tau-\int_{e}^{f} q_{2}(s, \sigma) g_{2}\left(x_{1}(s-\sigma)\right) d \sigma-h(s)\right| d s\right]-\Phi^{-1}[\Phi(\alpha) \\
& \left.\left.\quad+\int_{t}^{\infty} \frac{(s-t)^{n-1}}{(n-1) !}\left|\int_{c}^{d} q_{1}(s, \tau) g_{1}\left(x_{2}(s-\tau)\right) d \tau-\int_{e}^{f} q_{2}(s, \sigma) g_{2}\left(x_{2}(s-\sigma)\right) d \sigma-h(s)\right| d s\right]\right\} \leq \frac{1}{r(t)}\left\{p_{2}\left|x_{1}-x_{2}\right|\right. \\
& \quad+K \int_{t}^{\infty} \frac{(s-t)^{n-1}}{(n-1) !}\left[\int_{c}^{d} q_{1}(s, \tau)\left|g_{1}\left(x_{1}(s-\tau)\right)-g_{1}\left(x_{2}(s-\tau)\right)\right| d \tau\right. \\
& \left.\left.\quad+\int_{e}^{f} q_{2}(s, \sigma)\left|g_{2}\left(x_{1}(s-\sigma)\right)-g_{2}\left(x_{2}(s-\sigma)\right)\right| d \sigma\right] d s\right\} .
\end{aligned}
$$

Or, using (21),

$$
\begin{gathered}
\left|\left(T x_{1}\right)(t)-\left(T x_{2}\right)(t)\right| \leq\left|x_{1}-x_{2}\right| \frac{1}{2 p_{3}}\left\{p_{2}\right. \\
+K L \int_{t}^{\infty} \frac{(s-t)^{n-1}}{(n-1) !}\left[\int_{c}^{d} q_{1}(s, \tau) d \tau\right. \\
\left.\left.+\int_{e}^{f} q_{2}(s, \sigma) d \sigma\right] d s\right\}<\rho\left|x_{1}-x_{2}\right| .
\end{gathered}
$$

This implies with the sup norm that

$$
\left\|T x_{1}-T x_{2}\right\|<\rho\left\|x_{1}-x_{2}\right\|,
$$

and, taking (20) into account, we have

$$
\begin{aligned}
& (T x)(t) \geq \frac{1}{r(t)}\left\{-\int_{a}^{b} p(t, \theta) x(t-\theta) d \theta+\Phi^{-1}[\Phi(\alpha)\right. \\
& \left.\left.-\int_{t}^{\infty} \frac{(s-t)^{n-1}}{(n-1) !}\left[\int_{e}^{f} q_{2}(s, \sigma) g_{2}(x(s-\sigma)) d \sigma+h(s)\right] d s\right]\right\} \\
& \geq \frac{1}{2 p_{2}}\left\{-p_{2} M_{4}+\Phi^{-1}[\Phi(\alpha)\right. \\
& \left.\left.-\int_{t}^{\infty} \frac{(s-t)^{n-1}}{(n-1) !}\left(\beta_{2} \int_{e}^{f} q_{2}(s, \sigma) d \sigma+|h(s)|\right) d s\right]\right\} \geq M_{3} .
\end{aligned}
$$

These show that $T A \subset A$. Since $A$ is bounded, close, convex the $\Lambda$, in order to apply the contraction principle, we have to show that $T$ is a contraction mapping on $A$. For $\forall x_{1}, x_{2} \in A$, and $t \geq t_{1}$, 
From (9), one can choose $t_{1} \geq t_{0}+\mu$ and sufficiently large $t \geq t_{1}$ such that

$$
\begin{aligned}
& \int_{t}^{\infty} \frac{(s-t)^{n-1}}{(n-1) !}\left[\beta_{1} \int_{c}^{d} q_{1}(s, \tau) d \tau+|h(s)|\right] d s \\
& \quad \leq \Phi\left(\left(1+p_{4}\right) M_{6}\right)-\Phi(\alpha), \\
& \int_{t}^{\infty} \frac{(s-t)^{n-1}}{(n-1) !}\left[\beta_{2} \int_{e}^{f} q_{2}(s, \sigma) d \sigma+|h(s)|\right] d s
\end{aligned}
$$

$$
\leq \Phi(\alpha)-\Phi\left(-\frac{M_{5}}{p_{4}}\right)
$$

$$
\begin{aligned}
& \int_{t}^{\infty} \frac{(s-t)^{n-1}}{(n-1) !}\left[\int_{c}^{d} q_{1}(s, \tau) d \tau+\int_{e}^{f} q_{2}(s, \sigma) d \sigma\right] d s \\
& \quad \leq \frac{1+p_{4}}{K L}
\end{aligned}
$$

and define an operator $T$ on $A$ as follows:

$(T x)(t)$

$=\left\{\begin{array}{l}\frac{1}{r(t)}\left\{-\int_{a}^{b} p(t, \theta) x(t-\theta) d \theta+\Phi^{-1}\left[\Phi(\alpha)+\int_{t}^{\infty} \frac{(s-t)^{n-1}}{(n-1) !}\left[\int_{c}^{d} q_{1}(s, \tau) g_{1}(x(s-\tau)) d \tau-\int_{e}^{f} q_{2}(s, \sigma) g_{2}(x(s-\sigma)) d \sigma-h(s)\right] d s\right]\right\}, \quad t \geq t_{1}, \quad t_{0} \leq t \leq t_{1} \\ (T x)\left(t_{1}\right),\end{array}\right.$

It is easy to see that $T$ is continuous, for $t \geq t_{1}, x \in A$. By using (28), we have

$$
\begin{aligned}
& (T x)(t) \leq \frac{1}{r(t)}\left\{-\int_{a}^{b} p(t, \theta) x(t-\theta) d \theta+\Phi^{-1}[\Phi(\alpha)\right. \\
& \left.\left.+\int_{t}^{\infty} \frac{(s-t)^{n-1}}{(n-1) !}\left[\int_{c}^{d} q_{1}(s, \tau) g_{1}(x(s-\tau)) d \tau-h(s)\right] d s\right]\right\} \\
& \leq-p_{4} M_{6}+\Phi^{-1}[\Phi(\alpha) \\
& \left.+\int_{t}^{\infty} \frac{(s-t)^{n-1}}{(n-1) !}\left[\beta_{1} \int_{c}^{d} q_{1}(s, \tau) d \tau+|h(s)|\right] d s\right] \leq M_{6},
\end{aligned}
$$

and, taking (29) into account, we have

$$
(T x)(t) \geq \frac{1}{r(t)}\left\{-\int_{a}^{b} p(t, \theta) x(t-\theta) d \theta+\Phi^{-1}[\Phi(\alpha)\right.
$$

$$
\begin{aligned}
& \left|\left(T x_{1}\right)(t)-\left(T x_{2}\right)(t)\right| \leq \frac{1}{r(t)}\left\{\int_{a}^{b} p(t, \theta)\left|x_{1}(t-\theta)-x_{2}(t-\theta)\right| d \theta+\Phi^{-1}[\Phi(\alpha)\right. \\
& \left.\quad+\int_{t}^{\infty} \frac{(s-t)^{n-1}}{(n-1) !}\left|\int_{c}^{d} q_{1}(s, \tau) g_{1}\left(x_{1}(s-\tau)\right) d \tau-\int_{e}^{f} q_{2}(s, \sigma) g_{2}\left(x_{1}(s-\sigma)\right) d \sigma-h(s)\right| d s\right]-\Phi^{-1}[\Phi(\alpha) \\
& \left.\left.\quad+\int_{t}^{\infty} \frac{(s-t)^{n-1}}{(n-1) !}\left|\int_{c}^{d} q_{1}(s, \tau) g_{1}\left(x_{2}(s-\tau)\right) d \tau-\int_{e}^{f} q_{2}(s, \sigma) g_{2}\left(x_{2}(s-\sigma)\right) d \sigma-h(s)\right| d s\right]\right\} \leq \frac{1}{r(t)}\left\{-p_{4}\left|x_{1}-x_{2}\right|\right. \\
& \quad+K \int_{t}^{\infty} \frac{(s-t)^{n-1}}{(n-1) !}\left[\int_{c}^{d} q_{1}(s, \tau)\left|g_{1}\left(x_{1}(s-\tau)\right)-g_{1}\left(x_{2}(s-\tau)\right)\right| d \tau\right. \\
& \left.\left.\quad+\int_{e}^{f} q_{2}(s, \sigma)\left|g_{2}\left(x_{1}(s-\sigma)\right)-g_{2}\left(x_{2}(s-\sigma)\right)\right| d \sigma\right] d s\right\} .
\end{aligned}
$$

These show that $T A \subset A$. Since $A$ is bounded, close, convex subset of $\Lambda$, in order to apply the contraction principle, we have to show that $T$ is a contraction mapping on $A$. For $\forall x_{1}, x_{2} \in A$, and $t \geq t_{1}$,

Or, using (30),

$$
\begin{aligned}
& \left|\left(T x_{1}\right)(t)-\left(T x_{2}\right)(t)\right| \leq\left|x_{1}-x_{2}\right|\left(-p_{4}\right. \\
& \quad+K L \int_{t}^{\infty} \frac{(s-t)^{n-1}}{(n-1) !}\left[\int_{c}^{d} q_{1}(s, \tau) d \tau\right.
\end{aligned}
$$

$$
\left.\left.+\int_{e}^{f} q_{2}(s, \sigma) d \sigma\right] d s\right)<\rho\left|x_{1}-x_{2}\right|
$$

This implies with the sup norm that

$$
\left\|T x_{1}-T x_{2}\right\|<\rho\left\|x_{1}-x_{2}\right\|,
$$


where $\rho=1$, which shows that $T$ is a contraction mapping on $A$ and therefore there exists a unique solution, obviously a bounded positive solution of (1) $x \in A$, such that $T x=x$. The proof is complete.

Theorem 4. Assume that $-\infty<2 p_{5}<p_{6} \leq \int_{a}^{b} p(t, \theta) d \theta \leq$ $p_{5}<-1,-2 p_{5}<r(t)<-2 p_{6}$ and that (9) holds.

Then (1) has a bounded nonoscillatory solution.

Proof. Let $\Lambda$ be the set of all continuous and bounded functions on $\left[t_{0}, \infty\right)$ and the norm be $\|x(t)\|=\sup _{t_{0} \leq t<+\infty} x(t)$. Set $A=\left\{x \in \Lambda, M_{7} \leq x(t) \leq M_{8}, t \geq t_{0}\right\}$, where $M_{7}, M_{8}$ are two positive constants such that $-2 p_{6} M_{7}<\alpha<$ $\left(-2 p_{5}+p_{6}\right) M_{8}$. From (9), one can choose $t_{1} \geq t_{0}+\mu$ and sufficiently large $t \geq t_{1}$ such that

$$
\begin{aligned}
& \int_{t}^{\infty} \frac{(s-t)^{n-1}}{(n-1) !}\left[\beta_{1} \int_{c}^{d} q_{1}(s, \tau) d \tau+|h(s)|\right] d s \\
& \quad \leq \Phi\left(-2 p_{5} M_{8}+p_{6} M_{8}\right)-\Phi(\alpha) \\
& \int_{t}^{\infty} \frac{(s-t)^{n-1}}{(n-1) !}\left[\beta_{2} \int_{e}^{f} q_{2}(s, \sigma) d \sigma+|h(s)|\right] d s \\
& \quad \leq \Phi(\alpha)-\Phi\left(-2 p_{6} M_{7}\right), \\
& \int_{t}^{\infty} \frac{(s-t)^{n-1}}{(n-1) !}\left[\int_{c}^{d} q_{1}(s, \tau) d \tau+\int_{e}^{f} q_{2}(s, \sigma) d \sigma\right] d s \\
& \quad \leq \frac{p_{6}-2 p_{5}}{K L}
\end{aligned}
$$

and define an operator $T$ on $A$ as follows:

$(T x)(t)$

$=\left\{\begin{array}{l}\frac{1}{r(t)}\left\{-\int_{a}^{b} p(t, \theta) x(t-\theta) d \theta+\Phi^{-1}\left[\Phi(\alpha)+\int_{t}^{\infty} \frac{(s-t)^{n-1}}{(n-1) !}\left[\int_{c}^{d} q_{1}(s, \tau) g_{1}(x(s-\tau)) d \tau-\int_{e}^{f} q_{2}(s, \sigma) g_{2}(x(s-\sigma)) d \sigma-h(s)\right] d s\right]\right\}, \quad t \geq t_{1}, \quad \\ (T x)\left(t_{1}\right),\end{array}\right.$

It is easy to see that $T$ is continuous, for $t \geq t_{1}, x \in A$. By using (37), we have

$$
\begin{aligned}
& (T x)(t) \leq \frac{1}{r(t)}\left\{-\int_{a}^{b} p(t, \theta) x(t-\theta) d \theta+\Phi^{-1}[\Phi(\alpha)\right. \\
& \left.\left.\quad+\int_{t}^{\infty} \frac{(s-t)^{n-1}}{(n-1) !}\left[\int_{c}^{d} q_{1}(s, \tau) g_{1}(x(s-\tau)) d \tau-h(s)\right] d s\right]\right\} \\
& \quad \leq \frac{1}{-2 p_{5}}\left\{-p_{6} M_{8}+\Phi^{-1}[\Phi(\alpha)\right. \\
& \left.\left.\quad+\int_{t}^{\infty} \frac{(s-t)^{n-1}}{(n-1) !}\left[\beta_{1} \int_{c}^{d} q_{1}(s, \tau) d \tau+|h(s)|\right] d s\right]\right\} \leq M_{8},
\end{aligned}
$$

$$
\begin{aligned}
& \left|\left(T x_{1}\right)(t)-\left(T x_{2}\right)(t)\right| \leq \frac{1}{r(t)}\left\{\int_{a}^{b} p(t, \theta)\left|x_{1}(t-\theta)-x_{2}(t-\theta)\right| d \theta+\Phi^{-1}[\Phi(\alpha)\right. \\
& \left.\quad+\int_{t}^{\infty} \frac{(s-t)^{n-1}}{(n-1) !}\left|\int_{c}^{d} q_{1}(s, \tau) g_{1}\left(x_{1}(s-\tau)\right) d \tau-\int_{e}^{f} q_{2}(s, \sigma) g_{2}\left(x_{1}(s-\sigma)\right) d \sigma-h(s)\right| d s\right]-\Phi^{-1}[\Phi(\alpha) \\
& \left.\left.\quad+\int_{t}^{\infty} \frac{(s-t)^{n-1}}{(n-1) !}\left|\int_{c}^{d} q_{1}(s, \tau) g_{1}\left(x_{2}(s-\tau)\right) d \tau-\int_{e}^{f} q_{2}(s, \sigma) g_{2}\left(x_{2}(s-\sigma)\right) d \sigma-h(s)\right| d s\right]\right\} \leq \frac{1}{r(t)}\left\{-p_{6}\left|x_{1}-x_{2}\right|\right. \\
& \quad+K \int_{t}^{\infty} \frac{(s-t)^{n-1}}{(n-1) !}\left[\int_{c}^{d} q_{1}(s, \tau)\left|g_{1}\left(x_{1}(s-\tau)\right)-g_{1}\left(x_{2}(s-\tau)\right)\right| d \tau\right. \\
& \left.\left.\quad+\int_{e}^{f} q_{2}(s, \sigma)\left|g_{2}\left(x_{1}(s-\sigma)\right)-g_{2}\left(x_{2}(s-\sigma)\right)\right| d \sigma\right] d s\right\} .
\end{aligned}
$$


Or, using (39),

$$
\begin{gathered}
\left|\left(T x_{1}\right)(t)-\left(T x_{2}\right)(t)\right| \leq\left|x_{1}-x_{2}\right| \frac{1}{-2 p_{5}}\left\{-p_{6}\right. \\
+K L \int_{t}^{\infty} \frac{(s-t)^{n-1}}{(n-1) !}\left[\int_{c}^{d} q_{1}(s, \tau) d \tau\right. \\
\left.\left.+\int_{e}^{f} q_{2}(s, \sigma) d \sigma\right] d s\right\}<\rho\left|x_{1}-x_{2}\right| .
\end{gathered}
$$

This implies with the sup norm that

$$
\left\|T x_{1}-T x_{2}\right\|<\rho\left\|x_{1}-x_{2}\right\|,
$$

where $\rho=1$, which shows that $T$ is a contraction mapping on $A$ and therefore there exists a unique solution, obviously a bounded positive solution of (1) $x \in A$, such that $T x=x$. The proof is complete.

\section{Remark}

We note that when $n=1, r(t) \equiv 1, \Phi(u)=u^{\gamma}, q_{2}(t, \sigma)=$ 0 , and $h(t)=0$, we obtain (8). Thus, this paper great achievement was further obtained compared to that of [7].

\section{Example}

Example 1. Consider high-order neutral differential equation with distributed deviating arguments:

$$
\begin{aligned}
& {\left[\left(\frac{3}{2+\sin t} x(t)+\int_{\pi / 2}^{\pi} e^{-t} x(t-\theta) d \theta\right)^{3}\right]^{\prime \prime}} \\
& \quad+36 \int_{\pi}^{3 \pi / 2} \tau e^{-t} x(t-\tau) d \tau \\
& \quad-72 \int_{\pi / 2}^{\pi} \sigma e^{-2 t} x(t-\sigma) d \sigma=e^{-3 t}\left(15 \cos ^{3} t\right. \\
& \quad-30 \sin ^{3} t+63 \pi \sin ^{2} t-18 \pi \cos ^{2} t \\
& +\frac{1}{2} \sin ^{2} t\left(15 \cos t-27 \sin ^{2} t+33 \pi\right) \\
& \left.+6 \pi^{2}(\cos t-7 \sin t)+9 \pi^{3}\right)+18 e^{-2 t}(8 \pi \cos t \\
& \left.+\cos 2 t-\sin 2 t+4 \sin t-4 \cos t-3 \pi^{2}\right) \\
& +9 e^{-t}\left(4 \pi \cos t-6 \pi \sin t+5 \pi^{2}+2 \pi\right) .
\end{aligned}
$$

Here, $n=2, r(t)=3 /(2+\sin t), P(t, \theta)=e^{-t}, q_{1}(t, \tau)=$ $36 \tau e^{-t}, q_{2}(t, \sigma)=72 \sigma e^{-2 t}, \Phi(u)=u^{3}, g_{1}(u)=g_{2}(u)=u$, $a=e=\pi / 2, b=c=f=\pi, d=3 \pi / 2.1<r(t)=3 /(2+$ $\sin t)<3, \int_{\pi / 2}^{\pi} e^{-t} d \theta=(\pi / 2) e^{-t}<1, \int_{t_{0}}^{\infty} \int_{3 \pi / 2}^{\pi} 36 \tau s e^{-s} d \tau d s<$ $\infty, \int_{t_{0}}^{\infty} \int_{\pi / 2}^{\pi} \sigma s e^{-2 s} d \sigma d s<\infty, \int_{t_{0}}^{\infty} e^{-3 s} \mid 15 \cos ^{3} s-30 \sin ^{3} s+$ $63 \pi \sin ^{2} s-18 \pi \cos ^{2} s+(1 / 2) \sin 2 s\left(15 \cos s-27 \sin ^{2} s+33 \pi\right)$ $+6 \pi^{2}(\cos s-7 \sin s)+9 \pi^{3}\left|+18 e^{-2 s}\right| 8 \pi \cos s+\cos 2 s-\sin 2 s+$
$4 \sin s-4 \cos s-3 \pi^{2}\left|+9 e^{-s}\right| 4 \pi \cos s-6 \pi \sin s+5 \pi^{2}+2 \pi \mid d s<$ $\infty$.

Then it is easy to see that all the conditions of Theorem 1 are satisfied. In fact, $x(t)=2+\sin t$ is a nonoscillatory solution of (46).

\section{Competing Interests}

The authors declare that they have no competing interests.

\section{Acknowledgments}

This research is supported by Scientific Research Project Shanxi Datong University (no. 2011K3) and Scientific Research Startup Funding of Doctor of Shanxi Datong University (2015-B-07).

\section{References}

[1] Y. Zhou and B. G. Zhang, "Existence of nonoscillatory solutions of higher-order neutral differential equations with positive and negative coefficients," Applied Mathematics Letters, vol. 15, no. 7, pp. 867-874, 2002.

[2] W. Zhang, W. Feng, J. Yan, and J. Song, "Existence of nonoscillatory solutions of first-order linear neutral delay differential equations," Computers \& Mathematics with Applications, vol. 49, no. 7-8, pp. 1021-1027, 2005.

[3] Y. Yu and H. Wang, "Nonoscillatory solution of second-order nonlinear neutral delay equtions," Journal of Mathematical Analysis and Applications, vol. 311, pp. 445-456, 2005.

[4] T. Candan and R. S. Dahiya, "Existence of nonoscillatory solutions of first and second order neutral differential equations with distributed deviating arguments," Journal of the Franklin Institute, vol. 347, no. 7, pp. 1309-1316, 2010.

[5] T. Candan, "The existence of nonoscillatory solutions of higher order nonlinear neutral equations," Applied Mathematics Letters, vol. 25, no. 3, pp. 412-416, 2012.

[6] Y. J. Liu, J. W. Zhang, and J. R. Yan, "Existence of nonoscillatory solutions of higher order neutral differential equations with distributed deviating arguments," Acta Mathematicae Applicatae Sinica, vol. 38, no. 2, pp. 235-243, 2015 (Chinese).

[7] T. Candan, "Existence of nonoscillatory solutions of first-order nonlinear neutral differential equations," Applied Mathematics Letters, vol. 26, no. 12, pp. 1182-1186, 2013.

[8] T. Candan, "Existence of nonoscillatory solutions for system of higher order neutral differential equations," Mathematical and Computer Modelling, vol. 57, no. 3-4, pp. 375-381, 2013.

[9] Y. Liu, J. Zhang, and J. Yan, "Existence of nonoscillatory solutions for system of higher-order neutral differential equations with distributed deviating arguments," Discrete Dynamics in Nature and Society, vol. 2013, Article ID 391973, 8 pages, 2013.

[10] M. R. Kulenović and S. Hadžiomerspahić, "Existence of nonoscillatory solution of second order linear neutral delay equation," Journal of Mathematical Analysis and Applications, vol. 228, no. 2, pp. 436-448, 1998.

[11] Ö. Öcalan, "Oscillation of neutral differential equation with positive and negative coefficients," Journal of Mathematical Analysis and Applications, vol. 331, no. 1, pp. 644-654, 2007.

[12] I. Györi and G. Ladas, Oscillation Theory of Delay Differential Equations with Applications, Clarendon Press, Oxford, UK, 1991. 


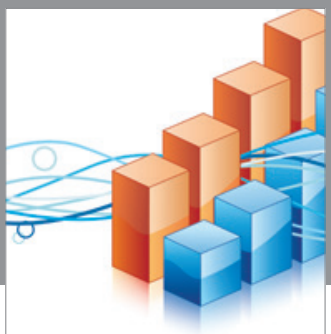

Advances in

Operations Research

vatem alat4

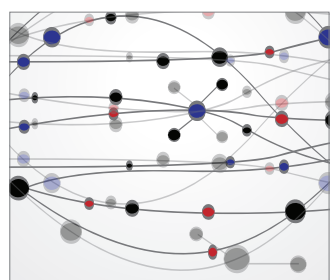

\section{The Scientific} World Journal
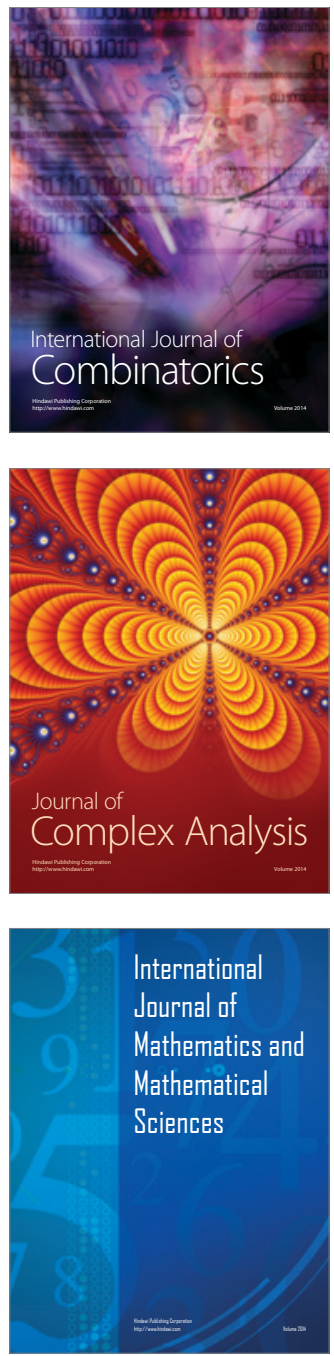
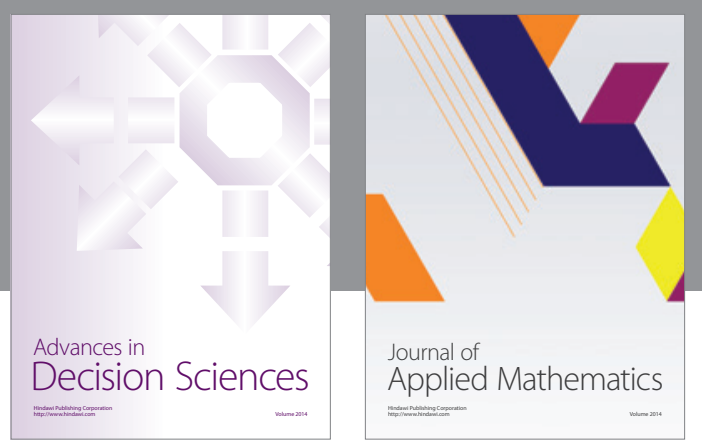

Algebra

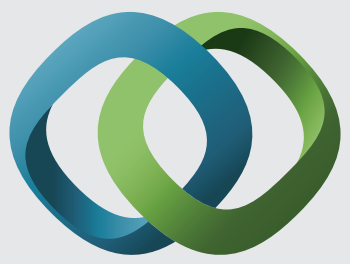

\section{Hindawi}

Submit your manuscripts at

http://www.hindawi.com
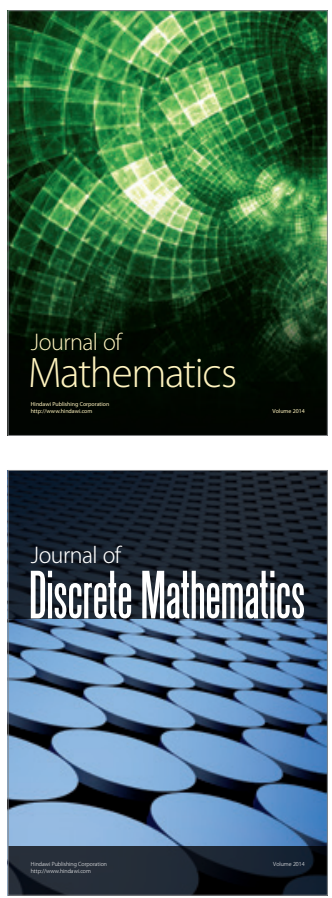

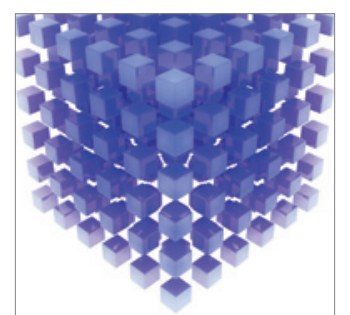

Mathematical Problems in Engineering
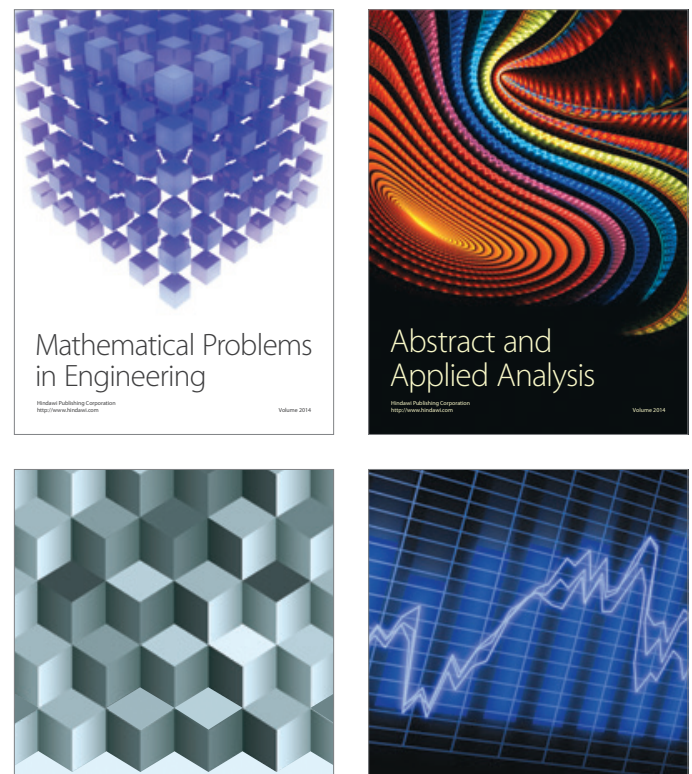

Journal of

Function Spaces

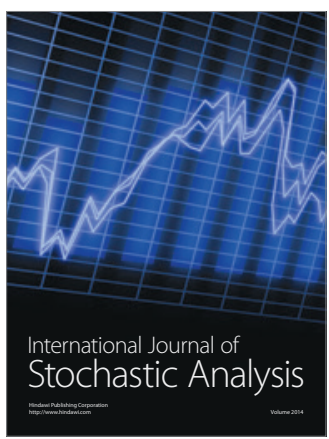

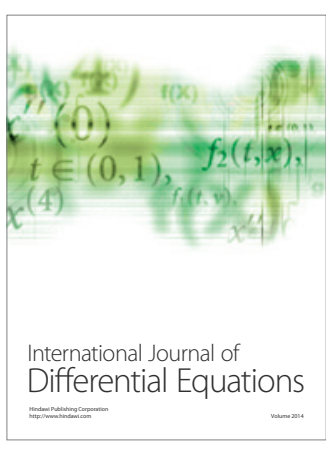
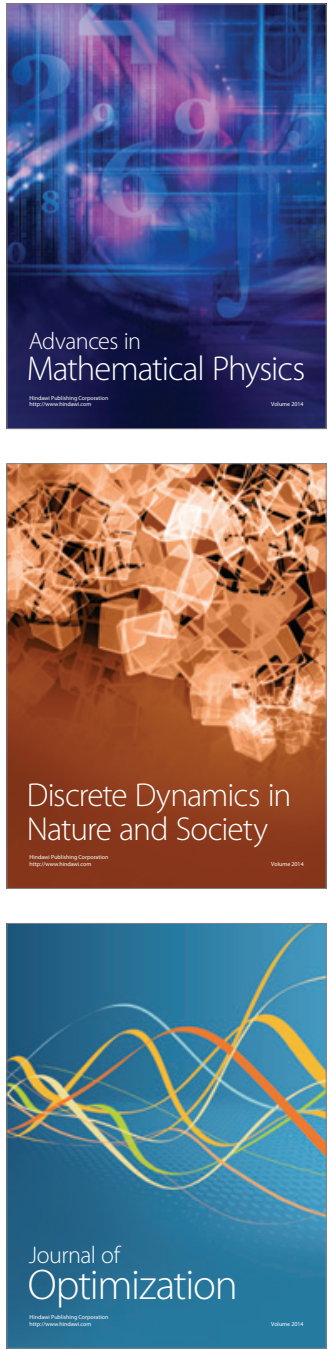